\title{
An experimental infection model to induce digital dermatitis infection in cattle
}

\author{
A. Gomez, ${ }^{\star 1}$ N. B. Cook, ${ }^{\star}$ N. D. Bernardoni, ${ }^{\star}$ J. Rieman, ${ }^{\star}$ A. F. Dusick, ${ }^{\star}$ R. Hartshorn, ${ }^{\star}$ M. T. Socha, $\dagger$ \\ D. H. Read,‡ and D. Döpfer* \\ ${ }^{*}$ School of Veterinary Medicine, University of Wisconsin, Madison 53706-1102 \\ †Zinpro Corporation, 10400 Viking Dr., Ste. 240, Eden Prairie, MN 55374 \\ $\ddagger$ California Animal Health and Food Safety Laboratory System, University of California-Davis, San Bernardino Branch 92408-2113
}

\section{ABSTRACT}

Bovine digital dermatitis (DD), also known as papillomatous digital dermatitis (foot warts), has been recognized as a major cause of lameness in cattle, with important economic and welfare consequences. The evaluation of therapeutic and preventive interventions aiming to control DD infections in dairy cattle is often challenged by the complex multifactorial etiology of the disease. An experimental infection model to induce acute DD lesions in a controlled environment is proposed. The goal was to provide a standard way of reproducing DD infections independent of external factors that could confound the natural course of the disease, such as management practices or infection pressure, resulting in transmission of DD between animals. A group of 4 yearling Holstein heifers free of any clinical evidence of hoof disease was recruited from a commercial dairy farm and housed in an experimental facility in 1 pen with slatted flooring. The hind feet were wrapped to mimic conditions of prolonged moisture (maceration) and reduced access to air (closure) and inoculated at the heel and dewclaw areas with a homogenate of a naturally occurring DD lesion skin biopsy or a culture broth of Treponema spp. After a period of 12 to $25 \mathrm{~d}, 4$ of 6 and 1 of 4 dewclaw areas inoculated with biopsied DD lesion or a Treponema spp. culture, respectively, had gross lesions compatible with DD. Histopathology confirmed the gross diagnosis in the sites inoculated with tissue homogenate. In the site inoculated with Treponema spp. culture broth, histopathology revealed an incipient DD lesion. Treponema spp. were detected by PCR in both naturally occurring DD homogenate and Treponema spp. culture broth inoculation sites. An experimental infection model to induce acute DD in cattle was developed, which may be used to evaluate interventions to control DD and study the pathogenesis of this infectious hoof disease in a controlled manner.

Received July 21, 2011.

Accepted December 20, 2011.

${ }^{1}$ Corresponding author: gomez3@wisc.edu
Key words: digital dermatitis, infection model, Treponema spp., cattle

\section{INTRODUCTION}

Bovine digital dermatitis (DD), also known as papillomatous digital dermatitis (PDD, foot warts), is a common, worldwide, painful, infectious disease of the feet of intensively managed cattle (Blowey and Sharp, 1988; Wells et al., 1999; Olechnowicz et al., 2010). Bovine $\mathrm{DD}$ is associated with large economic losses due to decreased reproductive performance and loss of milk production or increased risk for culling in addition to high treatment costs (Bruijnis et al., 2010; Cha et al., 2010; Ettema et al., 2010). Changes in behavior (Cook and Nordlund, 2009; Pavlenko et al., 2009; Gomez and Cook, 2010) and discomfort (Flower and Weary, 2009) associated with lameness caused by DD result in a major impact on animal welfare. Digital dermatitis is characterized by circumscribed ulceroproliferative lesions involving the skin near the interdigital cleft at the plantar aspect of the hind feet (Read and Walker, 1994; Blowey and Sharp, 1988; Read and Walker, 1998b).

The cause of DD is multifactorial with an essential spirochetal bacterial component (Read et al., 1998). The interaction of causative factors, including spirochetes (Walker et al., 1995), the host (Scholey et al., 2010), and the environment (Rodríguez-Lainz et al., 1996; Wells et al., 1999), result in DD. Sequential histopathological, immunohistochemical, and ultrastructural studies of experimental PDD confirmed that spirochetes (Read et al., 1998) play an important role in the pathogenesis of DD (Walker et al., 1995). Several cultural, phenotypic and molecular studies have demonstrated that the spirochetes belong to a diverse phylogenetic group of Treponema spp. (Walker et al., 1998; Evans et al., 2008; Nordhoff et al., 2008). Other bacteria isolated or identified by molecular techniques have included Porphyromonas levii, an unidentified, anaerobic, non-motile, long, straight, gram-negative rod with pointed ends, an untyped Mycoplasma sp. and Campylobacter fecalis (Döpfer et al., 1997; Berry et al., 
2010; Yano et al., 2010). The pathogenic significance of the other isolates is unknown but, because bacterial morphotypes other than spirochetes are inconsistently present and are rarely seen to invade viable tissue in DD lesions, they may not play an important pathogenic role. Diagnosis of active DD is based on histopathologic criteria, namely, loss of stratum corneum and granulosum, exposure of stratum spinosum or dermis, invasion of stratum spinosum or dermis by spirochetes, and reactive inflammation (Read and Walker, 1994). Risk factors for increased prevalence of DD include freestall herds compared with tie-stall herds (Sogstad et al., 2005), high moisture, chemical or physical trauma of the digital skin, purchase of infected cattle, and muddy unhygienic environments (Rodríguez-Lainz et al., 1996; Wells et al., 1999). The genetic makeup of the host is currently receiving some attention as a possible risk factor for DD (Scholey et al., 2010). The evaluation of interventions aimed at prevention and control of DD under field conditions can often be confounded due to the variability and complexity of the interaction between the host, pathogens, and the environment. This has resulted in variable observed effects of therapy and prevention at an individual and group level (Speijers et al., 2010; Teixeira et al., 2010). The current study was designed to establish an experimental infection model to induce acute DD lesions in a controlled environment to provide a model for quantifying the potential effect of therapeutic and preventive measures for the control of DD.

\section{MATERIALS AND METHODS}

\section{Experimental Animals}

Four Holstein heifers (A, B, C, and D), 14 to 16 mo of age and weighing between 270 and $402 \mathrm{~kg}$ were purchased from a commercial dairy farm in Wisconsin. At the start of the study in January 2010, all 4 heifers had no clinical signs of hoof disease. Upon arrival at the Livestock Laboratory at the University of Wisconsin-Madison, the heifers received a prophylactic dose of Ceftiofur $(6.6 \mathrm{mg} / \mathrm{kg}$ of Excede; Pfizer Inc., New York, NY) and were vaccinated according to label directions against bovine virus diarrhea, infectious bovine rhinotracheitis and parainfluenza 3 (Bovi-Shield Gold 4; Pfizer Inc.), and Clostridium spp. (Vision 7; Intervet Inc., Summit, NJ). Health status was recorded daily for appetite, rectal body temperature, and heart and respiratory rate and twice daily for lameness (differences in weight-bearing between rear legs) or discomfort (constant change of weight distribution or increased bout behavior in relation to the group) in 0.5 to 1 -h frames. Body weight was measured twice per month. The ani- mals were housed in a pen $\left(40 \mathrm{~m}^{2}\right)$ with a slatted floor surface that was hose cleaned once per day and automatic water flushed 6 times per day. Ventilation was provided by means of a positive-pressure ventilation system. Total mixed ration was composed of haylage, corn silage, and a standard concentrate supplement and delivered once per day according to the NRC specifications for an average daily gain of $0.7 \mathrm{~kg} / \mathrm{d}$ (NRC, 2001).

The study was designed to have a maximum duration of $60 \mathrm{~d}$ and the Animal Care and Use Protocol No. V-1429 was approved by the Research Animal Resources Center of the University of Wisconsin-Madison.

\section{Biopsy Technique for Collection of Inoculum}

To generate inocula for the experimental infections, homogenized skin biopsies from active DD lesions from 5 naturally infected donor cows were used. The donor cows were recruited from 1 commercial dairy farm and were considered as donors if they had an acute ulcerative DD lesion of more than $20 \mathrm{~mm}$ in diameter. Before the biopsy, 3 s.c. injections of 2 to $3 \mathrm{~mL}$ of lidocaine (Lidocaine $2 \%$; Hospira Inc., Lake Forest, IL) were used for local anesthesia. The lesions were cleaned gently with a brush, rinsed with tap water, and dried using a disposable paper towel. Sterile technique and a \#20 scalpel blade (Becton, Dickinson and Co., Franklin Lakes, NJ) were used to shave off the outer superficial $2 \mathrm{~mm}$ of the DD lesion. In this way, the epidermal layer of the acute lesion was completely removed. Biopsies were rinsed with sterile PBS (Invitrogen Corp., Carlsbad, CA) and placed in sterile 15-mL conical tubes (Falcon; Becton, Dickinson and Co.) containing sterile oral treponeme enrichment broth (OTEB; Anaerobe Systems, Morgan Hill, CA). The tubes were supplemented with $5 \mu \mathrm{g} / \mathrm{mL}$ of rifampicin (Sanofi-Aventis, Bridgewater, NJ) and 5 $\mu \mathrm{g} / \mathrm{mL}$ of enrofloxacin (Bayer, Leverkusen, Germany), aiming to reduce the extra growth of nondesirable organisms during transport. The tubes were transported to the laboratory at room temperature and processed within $2 \mathrm{~h}$ of collection.

\section{Preparation of the Inocula}

Two types of inocula were used to induce the experimental DD infections in the 4 heifers: a homogenate of the skin biopsy, and a Treponema spp. clonal isolate culture broth of $10^{8} \mathrm{cfu} / \mathrm{mL}$. The homogenate was made by grinding the biopsy material (as a mixture of all biopsy homogenates from the donor cows) in a sterile mortar in the presence of $3 \mathrm{~mL}$ of OTEB. Approximately $5 \mathrm{~g}$ of the resulting homogenate was transferred into a sterile 50-mL conical tube and OTEB was added to reach a volume of $2 \mathrm{~mL}$ of inoculum per 

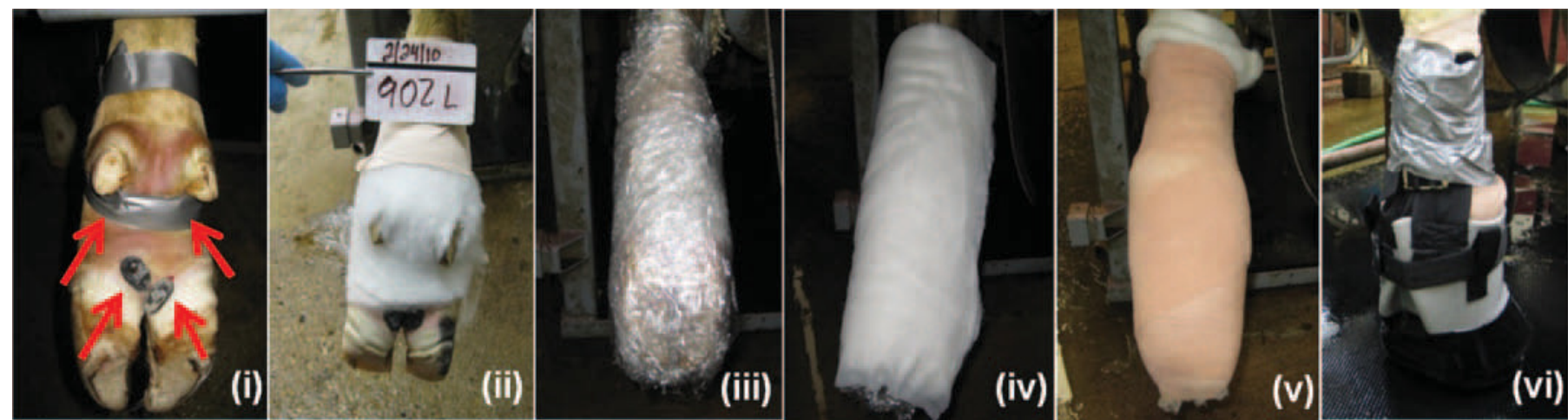

Figure 1. Layers of the foot bandages used to induce experimental digital dermatitis (DD) infections in the 4 heifers (i-iv); (i) the location and fixation of the rubber reservoirs containing $0.5 \mathrm{~mL}$ of inoculum each (red arrows), (ii) $50-\times 18$-cm cotton sheet; (iii) polyethylene (Saran) wrap; (iv) 100- × 100-cm cotton sheet; (v) self-adherent wrap; (vi) rubber boot.

foot inoculated. To improve the anaerobic condition of the inoculum, 1 drop of Oxyrase solution (30 units/ $\mathrm{mL}$; Oxyrase Inc., Mansfield, $\mathrm{OH}$ ) for every $5 \mathrm{~mL}$ of inoculum was added according to manufacturer's specifications. A wet mount was made using about $25 \mu \mathrm{L}$ of the inoculum and examined by dark-field microscopy at $20 \times(80 \mathrm{i}$; Nikon Inc., Milwaukee, WI) for motile spirochetes. Upon finding an estimated count of $>1,000$ spiral, motile microorganisms per $20 \times$ field, the inoculum was considered to have sufficient potency for infection. Polymerase chain reaction confirmation (Dreher et al., 1999; Evans et al., 2008) for the presence of Treponema spp. was performed in a subsample of every biopsy with a positive result in all cases.

The Treponema spp. culture was prepared from treponemes isolated from acute DD lesions in cattle from naturally occurring acute DD lesions in cattle from a commercial farm in Wisconsin. The isolates obtained from single-colony selection were grown to log phase in OTEB and before inoculation were confirmed to contain Treponema spp. using PCR reported by Evans et al. (2009). The conventional PCR reported by Seshadri et al. (2004) revealed the isolate to be a Treponema vincentii subtype.

\section{Inoculation Sites, Technique of Inoculation, and Wrapping Procedure}

A combination of prolonged moisture (maceration) and reduced access to air (closure) was found to be an essential precondition for experimental PDD infections (Read and Walker, 1998a). Accordingly, the rear legs of all 4 heifers were wrapped for $18 \mathrm{~d}$ before inoculation from $10 \mathrm{~cm}$ distal to the hock down to the toe to achieve skin closure and maceration using the following 6-step procedure (Figure 1):
1) Four skin sites per leg were used for placing the inocula: 2 at the distal coronary band of the dewclaws and 2 at the skin-horn junction in the axial aspect of the heel, close to the interdigital cleft [Figure 1(i); red arrows]. A volume of 0.5 $\mathrm{mL}$ of the inoculums was placed into each reservoir. Each inoculum was contained in the infection site using a 1-mL rubber reservoir composed of an oval-shaped rubber base attached to an oval-shaped rubber ring that was placed onto the skin surface and secured with strips of duct tape at the prescribed heel and dewclaw areas. Reservoirs at the heel area were additionally glued to the skin (Super Glue; Henkel Co., Westlake, $\mathrm{OH})$ to provide appropriate contact of reservoir to skin, which cannot be achieved well with duct tape strips alone.

2) A 50- $\times 18-\mathrm{cm}$ piece of cotton sheet (Jorgensen Labs Inc., Loveland, CO) was fixed with Elastikon (Johnson and Johnson Services Inc., Langhorne, PA) above the dewclaw area. Two holes were made at the dewclaw level, allowing the dewclaws to protrude through the material.

3) Seven layers of polyethylene wrap (Saran; SC Johnson \& Son Inc., Racine, WI) were used to cover the leg from the tip of the toes to $10 \mathrm{~cm}$ below the hock.

4) Three tap water-soaked $100 \times 100-\mathrm{cm}$ cotton sheets were rolled around the leg, fixed with Elastikon at the top of the bandage and wrapped with a roll of self-adherent wrap (Coban; 3M Co., St. Paul, MN) while avoiding pressure to the dewclaws.

5) A plastic boot (EasySoaker; Easy Care Inc., Tucson, AZ) was attached to the foot using customized straps. 
6) Finally, the bandage left uncovered by the boot was wrapped with duct tape (3M Performance Plus Duct Tape 8979; 3M Co.). The boots were filled with tap water twice per day at 12 -h intervals. All procedures were performed using a standing hoof trimming chute (H-Series; Comfort Hoof Care Inc., Baraboo, WI).

\section{Timeline for the Experimental Infections and Clinical Evaluation of the Skin}

For $7 \mathrm{~d}$ after arrival to the experimental facility, the 4 heifers were allowed to adapt to the new environment without handling except for the vaccinations and antimicrobial treatments described above. A closure and maceration period followed for a period of $18 \mathrm{~d}$, during which the rear feet were wrapped using the technique described. After the closure and maceration period, the inoculum was applied on d 18 of the study (first inoculation) and on d 45 (second inoculation) with the following sequence: during this first inoculation, heifer A was inoculated using biopsy homogenate in both rear legs; $\mathrm{B}$ and $\mathrm{C}$ were inoculated in the right rear leg using the biopsy homogenate and using the Treponema spp. culture in the left rear leg. Both legs in heifer D were used as sham controls, and the rubber reservoirs were placed without inoculum. During the second inoculation on d 45, heifer B (which did not show any lesions during the following $27 \mathrm{~d}$ after the first inoculation) was reinoculated with biopsy homogenate in the right rear leg and with Treponema spp. culture in the left rear leg, and heifer D (which was used as a sham control during the first inoculation) was also inoculated with biopsy homogenate in the right rear leg and with Treponema spp. culture in the left rear leg. At first inspection $7 \mathrm{~d}$ after inoculation (d 25 and 52 of the study), the reservoirs were removed from the inoculation sites. The feet were examined at intervals of 3 or $7 \mathrm{~d}$ post-inoculation as shown in the timeline (Figure 2). During each of the evaluations, bandages were removed, pictures of the inoculation sites were taken, and the skin at these sites was neither touched nor cleaned. Clinical evaluation of the skin was performed based on visual degree of s.c. edema, coloration, and presence of lesions, and observable reaction to the touch, presence of skin peeling and skin softness variation. Digital dermatitis lesions were measured, their macroscopic appearance recorded, and the bandages were replaced when no DD lesions were found or when they were smaller than $20 \mathrm{~mm}$ across their greatest dimension. When a lesion greater than 20 $\mathrm{mm}$ was found, that determined the termination of the bandaging process for the leg. This met the standard of an acute M2 lesion using the classification system developed by Döpfer et al. (1997). At the termination of the study, all lesions were confirmed histopathologically. After meeting the inclusion criterion of $20 \mathrm{~mm}$ across the greatest dimension for an acute experimental DD lesion (or due to the termination of the study for heifer B right rear leg with a lesion $12 \mathrm{~mm}$ in size) a skin biopsy was taken using the standardized technique as described below, and the lesion treated with $10 \mathrm{~mL}$ of liquid oxytetracycline (Oxy-Mycin $100 \mathrm{mg} /$ mL; Agripharm Products, Westlake, TX) under a light wrap. After topical treatment, the leg was excluded from the study and inspected for healing $1 \mathrm{wk}$ after treatment. For the first $46 \mathrm{~d}$ of the trial, 1 of the animals (heifer D) was used as a sham control, following the prescribed protocol as per the 3 principals, except that no inoculum was placed. Because active DD lesions were identified in heifers $\mathrm{A}$ and $\mathrm{C}$ before $\mathrm{d} 45$ of the study, those heifers were excluded from the second inoculation but kept in the group to monitor the DD lesion evolution.

\section{Sampling Technique}

After macroscopic identification of a lesion resembling clinical DD, 2 biopsies were taken under local anesthesia using a True-Cut disposable punch biopsy 6 $\mathrm{mm}$ in diameter (Robbins Instruments Inc., Chatham, NJ) after gentle brushing and rinsing the lesion surface with tap water. One biopsy was placed in a $4-\mathrm{mL}$ sterile tube containing $2 \mathrm{~mL}$ of OTEB and submitted at room temperature for culture of spirochetes. The second punch biopsy was performed and the skin plug was placed in a $5 \%$ phosphate buffered formaldehyde solution and submitted for histopathological evaluation. Additional biopsies were taken from control legs and at the end of the study from legs with no macroscopic DD lesions diagnosed.

\section{Histopathology}

Formalin-fixed biopsies were routinely paraffin embedded, sectioned at 4- $\mu \mathrm{m}$ thickness and stained with hematoxylin-eosin, May-Grünwald-Giemsa, modified Steiner silver and a histochemical stain using a polyclonal antiserum to a PDD-associated Treponema spp. (Walker isolate 1-9185MED; Walker et al., 1997). Biopsies were classified according to prescribed histopathologic guidelines (Berry et al., 2010). The classification was blind to clinical and macroscopic appearance, and treatment (site and type of inoculation) assignment. Histopathologic categories were DD, incipient DD, nonspecific dermatosis or dermatitis (absence of 


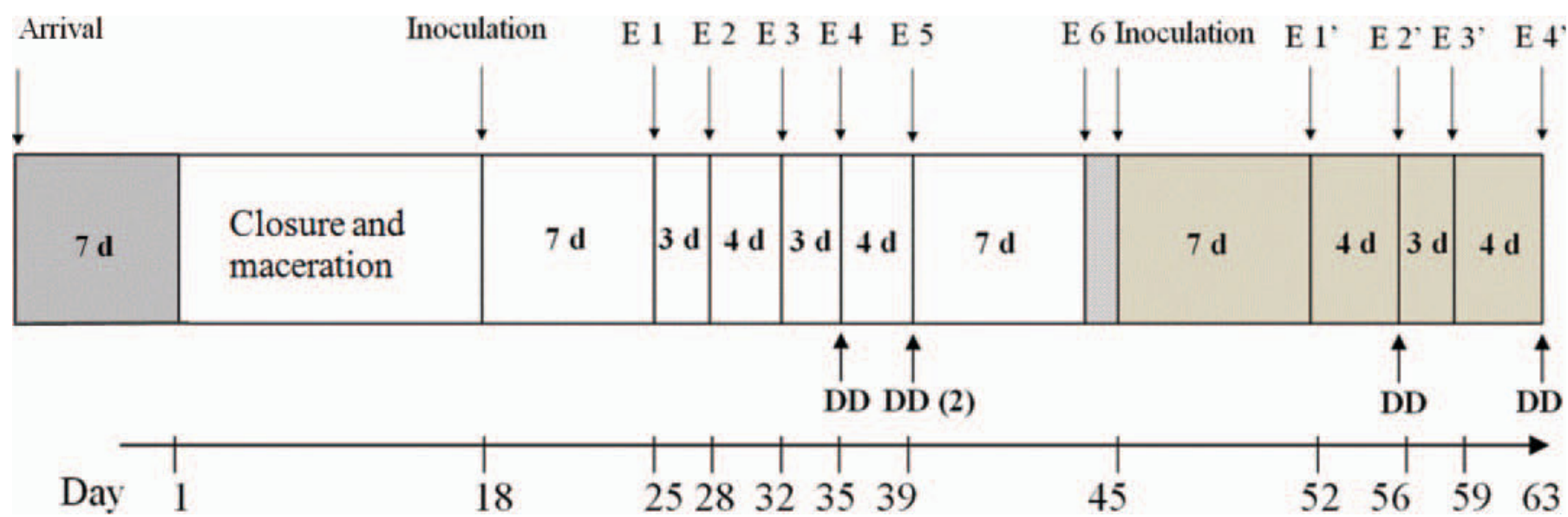

Figure 2. Timeline of the experiment, showing periods of pre-inoculation closure and maceration and inoculation days, evaluation (E) number, interval days between events, and when digital dermatitis diagnosis $[\mathrm{DD}(\#)]$ was made. Color version available in the online PDF.

typical signs of inflammation and profuse infiltration of neutrophils, respectively), and normal skin. Histopathologic criteria essential for a diagnosis of DD were, namely, exposure of stratum spinosum or ulceration of dermis, invasion of stratum spinosum or dermis by Treponema spp.-dominant bacterial mat, and reactive inflammation. In addition, DD biopsies were classified according to prominence of spirochetal and non-spirochetal organisms within the bacterial mat by arbitrary grading ranging from $1+[$ rare $=2-3 /$ hpf, where hpf $=$ high-power field in relation to the area visible under the maximum magnification power $(400 \times)$ of the objective] to $4+$ (dense mat; Figure 3), and according to location relative to the bacterial mat thickness (surface $=$ depth of $50 \mu \mathrm{m}$; deep = depth of $500 \mu \mathrm{m}$ ). Criteria for incipient DD were, namely, the same as for DD except that the Treponema spp.-dominant bacterial mat was sparse, invasion by spirochetes was slight, no ulceration was present, and reactive inflammation consisted of small numbers of mononuclear leucocytes in the dermis.

\section{Dark-Field Microscopy and PCR Determination}

A wet mount (approximately $10 \mu \mathrm{g}$ of biopsy homogenate and a drop of distilled water) was made at room temperature of all biopsies and examined under a dark-field microscope at 20× (Nikon Eclipse 80i; Nikon Instruments Inc., Melville, NY) for motile spirochetes within minutes of the biopsy collection. Confirmation of Treponema spp. microorganisms by PCR was performed using a procedure modified according to Evans et al. (2008) as follows: genomic DNA (gDNA) was extracted by heat lysis using the PrepMan Ultra kit (Applied Biosystems, Fountain City, CA). The PrepMan Ultra heat lysis protocol for gDNA extraction was performed as follows: approximately $100 \mu \mathrm{L}$ of minced biopsied tissue collected in $0.9 \mathrm{~mL}$ of OTEB media was centrifuged for 5 min at $13,400 \times g$ and the supernatant was discarded. The remaining pellet was resuspended in $100 \mu \mathrm{L}$ of PrepMan Ultra Sample Preparation Reagent and vortexed vigorously. The suspension was incubated on a dry heat block for $10 \mathrm{~min}$ at $100^{\circ} \mathrm{C}$, followed by cooling to $22^{\circ} \mathrm{C}$ for $2 \mathrm{~min}$, and was then centrifuged for 3 min at $13,400 \times g$. Fifty microliters of the resulting supernatant (DNA template) was transferred to a labeled microcentrifuge tube and stored at $-20^{\circ} \mathrm{C}$ for the PCR.

The quantitative real-time PCR assay was performed in $25-\mu \mathrm{L}$ reactions containing $2.5 \mu \mathrm{L}$ of extracted gDNA template, $12.5 \mu \mathrm{L}$ of $2 \times$ QuantiTect Multiplex PCR NoROX Master Mix (Qiagen GmbH, Hilden, Germany), $1 \mu \mathrm{L}$ of a primer-probe mix (forward primer 5'-CAAGGCDWYGATGGGTAT-3', reverse primer 5'-GTCAGACTTYCGTCCATTG-3', probe FAM 5'-CGGACACATTGGGACTGAGATACG-3' BHQ1) containing a final primer concentration of $0.4 \mu M$ and final probe concentration of $0.1 \mu M$ and sterile PCRgrade water. The amplicon identifies the isolates as Treponema spp. and the protocol is modified from Evans et al. (2008). The PCR reactions were performed on an MX3005P qPCR system (Agilent Technologies Inc., Santa Clara, CA) using the detection channel FAM (6-carboxyfluorescein). The PCR protocol consisted of an initial activation at $95^{\circ} \mathrm{C}$ for $10 \mathrm{~min}$, followed by 40 cycles of $95^{\circ} \mathrm{C}$ for $1 \mathrm{~min}$ and $60^{\circ} \mathrm{C}$ for $1 \mathrm{~min}$. Real-time fluorescence data acquisition occurred at the end of each annealing phase. The threshold fluorescence was $1,628 \mathrm{dR}$ (the baseline subtracted fluorescence reading). Cycle threshold (Ct) values were cut off at 38 . Standard curves of the $\mathrm{Ct}$ values were plotted against 


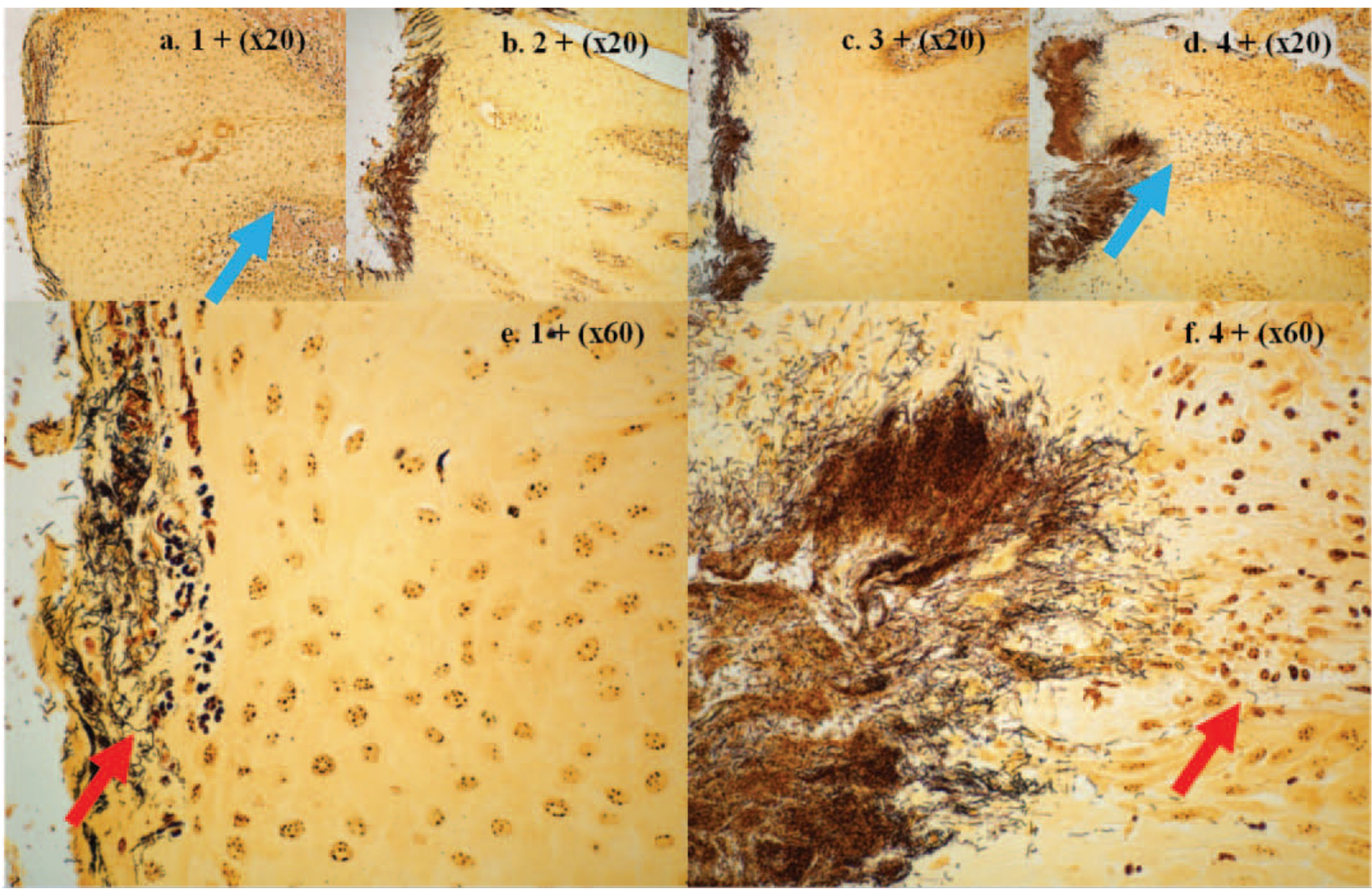

Figure 3. Example of grading of prominence of spirochetes in the bacterial mat using Steiner silver stain. a and e) grade $1+(5 \mu \mathrm{m} ; \times 20$ and $\times 60)$; b) grade $2+(\times 20)$; c) grade $3+(\times 20)$; $d$ and $\mathrm{f})$ grade $4+(500 \mu \mathrm{m} ; \times 20$ and $\times 60)$. Red arrows $=$ spirochetal organisms; blue arrows $=$ papillary dermis.

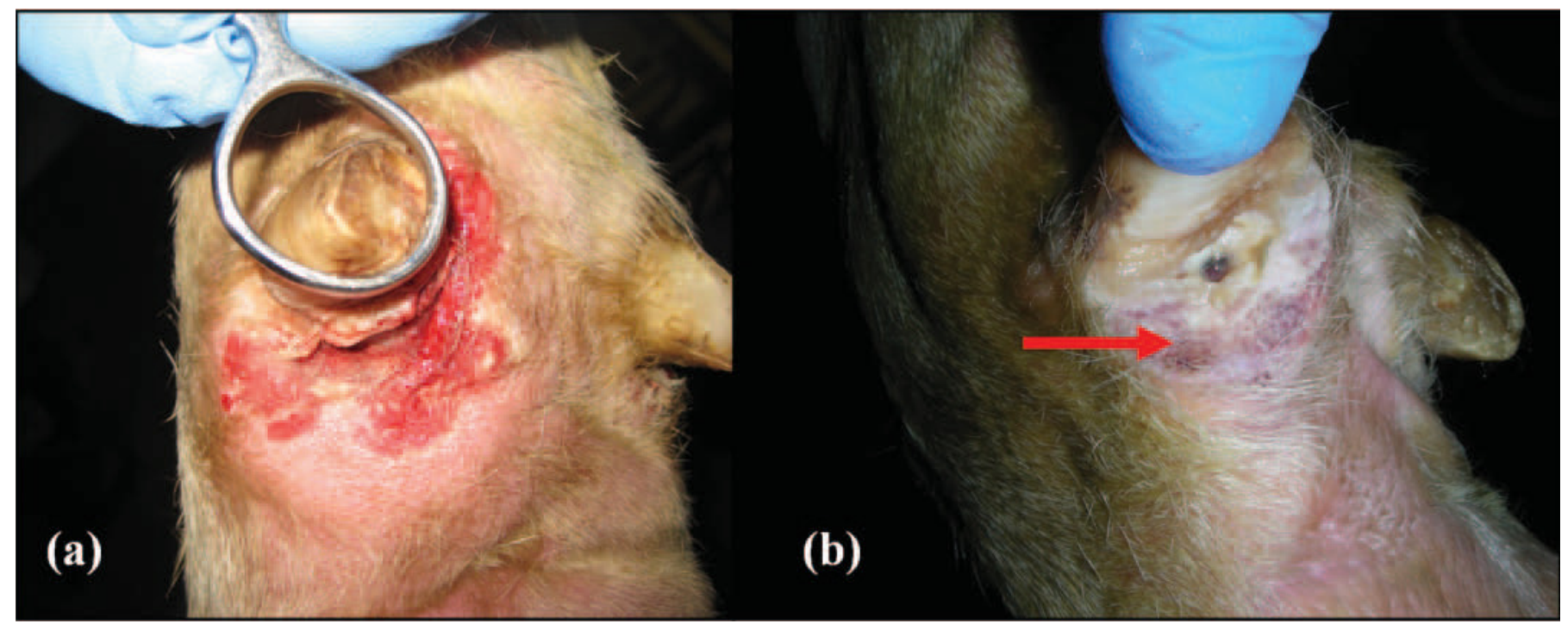

Figure 4. a) Digital dermatitis (DD) lesion at dewclaw area induced using tissue homogenate inoculum from naturally occurring DD (heifer A, right rear leg, $21 \mathrm{~d}$ post-infection); b) DD lesion at dewclaw area (red arrow) induced using a Treponema spp. culture broth (heifer C, left rear leg, $21 \mathrm{~d}$ post-inoculation). 
Table 1. Clinical outcome of experimental inoculation of skin adjacent to dewclaws of rear legs of 4 Holstein heifers (heifers B and D were reinoculated $28 \mathrm{~d}$ after the first inoculation)

\begin{tabular}{llll}
\hline $\begin{array}{l}\text { Heifer } \\
\text { identification }\end{array}$ & Foot $^{1,2}$ & $\begin{array}{l}\text { Inoculum }_{\text {type }^{3}} \\
\text { A }\end{array}$ & $\begin{array}{l}\text { Histopathologic } \\
\text { diagnosis }\end{array}$ \\
\hline $\mathrm{RR}$ & Tissue homogenate & DD \\
$\mathrm{B}^{4}$ & $\mathrm{LR}$ & Tissue homogenate & DD \\
$\mathrm{B}^{4}$ & $\mathrm{RR}$ & Tissue homogenate & ND \\
$\mathrm{B}$ & $\mathrm{LR}$ & Treponema culture & ND \\
$\mathrm{B}$ & $\mathrm{RR}$ & Tissue homogenate & ND \\
$\mathrm{C}$ & $\mathrm{LR}$ & Treponema culture & DD \\
$\mathrm{C}$ & $\mathrm{RR}$ & Tissue homogenate & ND \\
$\mathrm{D}$ & $\mathrm{LR}$ & Treponema culture & ND \\
$\mathrm{D}$ & $\mathrm{RR}$ & Tissue homogenate & ND \\
$D^{6}$ & $\mathrm{LR}$ & Treponema culture & ND \\
$D$ & $R R$ & No inoculum & No inoculum \\
\hline
\end{tabular}

${ }^{1} \mathrm{RR}=$ right rear leg; $\mathrm{LR}=$ left rear leg.

${ }^{2}$ All of the infections were diagnosed at the dewclaws and none of them at the heel inoculation sites.

${ }^{3}$ Tissue homogenate was prepared from naturally occurring bovine digital dermatitis (DD) lesions. Treponema spp. culture was prepared from a clonal isolate obtained from a subculture of a naturally occurring DD lesion culture.

${ }^{4}$ Heifer B second inoculation.

${ }^{5} \mathrm{ND}=$ histopathology not done.

${ }^{6}$ Last 2 rows represent the animal with the sham inoculation during the first inoculation (heifer D).

the concentration of DNA in the sample, as determined by UV spectroscopy (NanoDrop 2000C; Thermo Scientific, Wilmington, DE).

\section{RESULTS}

\section{Results of Experimental Infections}

Bovine DD was confirmed histopathologically in 4 of 6 of the legs inoculated with a fresh DD biopsy homogenate and in 1 of 4 of legs inoculated with Treponema spp. broth culture incipient DD. Reproduced DD lesions were observed exclusively in the dewclaw areas. None of the animals showed symptoms of discomfort such as lameness or general depression due to the closure and maceration procedures. None of the legs was diagnosed as lame and behavior was considered normal, with an average daily weight gain of $0.86 \mathrm{~kg}$ recorded during the experimental period. The results of the experimental inoculations performed in this study are summarized in Table 1. All of the experimental DD lesions had observable spirochetes by dark-field microscopy, Treponema spp. by PCR, and invasive spirochetes by Steiner silver stain (Figure 3; Table 2) and immunohistochemistry

Table 2. Summary of histopathologic findings in biopsies of dewclaw skin of rear legs of 3 Holstein heifers inoculated with bovine digital dermatitis (DD) tissue homogenate or Treponema spp. culture

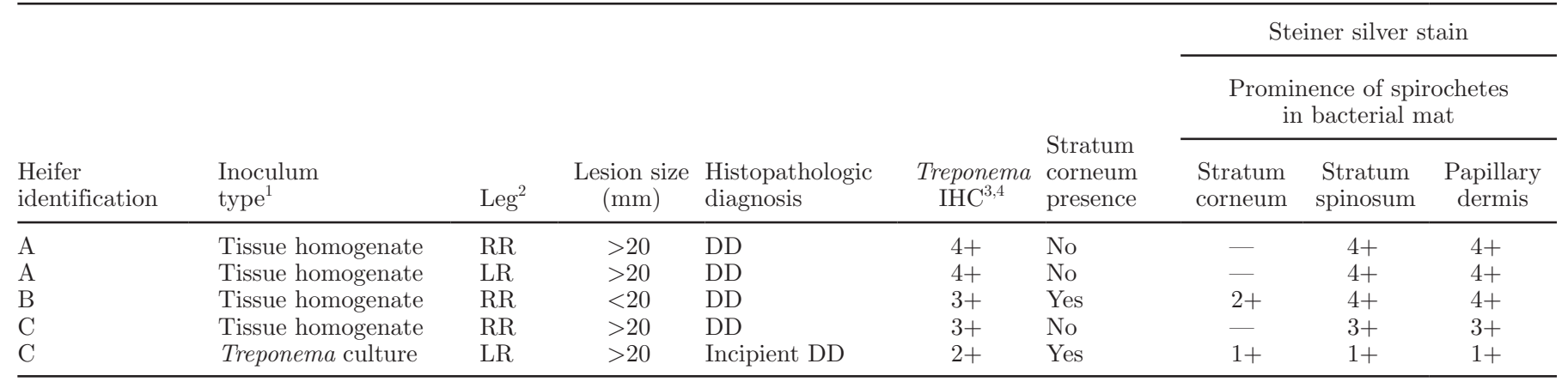

${ }^{1}$ Tissue homogenate was prepared from naturally occurring DD lesions. Treponema spp. culture was prepared from a clonal isolate obtained from a subculture of a naturally occurring DD lesion culture.

${ }^{2} \mathrm{RR}=$ right rear leg; $\mathrm{LR}=$ left rear leg.

${ }^{3} \mathrm{IHC}=$ immunohistochemistry.

${ }^{4} 1+$ to $4+=$ arbitrary grading of prominence of spirochetes from $1+[$ rare $=2-3 /$ hpf, where hpf $=$ high-power field in relation to the area visible under the maximum magnification power $(400 \times)$ of the objective] to $4+$ (profuse $=$ dense mat). 
(Table 2). Treponema spp. isolates were recovered from all DD lesions reproduced during the experimental infection. However, negative results were obtained for dark-field microscopy, histopathology, and culture from the control legs and from legs with no macroscopic DD lesions diagnosed.

\section{Clinical Evaluation of the Skin}

Changes in the skin subjected to closure and maceration were characterized by s.c. edema, slight accumulation of sebum, fetid smell, and softening of the corneal tissue. These changes were found throughout the study period, most notably at evaluation d 18 and 25 (Figure 2) most likely due to the inoculation process. Slightly more pronounced skin modifications were observed at the distal aspect of the dewclaws' coronary bands. The sites of inoculation showed variable degrees of maceration and signs of initial skin degradation that developed to healthy skin approximately $14 \mathrm{~d}$ after the initial inoculation (study d 32; Figure 2) in legs where DD were not diagnosed. The macroscopic appearance of the skin at the sites of sham inoculation in the control animal showed minor skin changes after inoculation.

All lesions were diagnosed along the distal area adjacent to the coronary band of the dewclaws. Initially, at post-infection d 7 to 18 , a variable number (1 to 3 ) of round, smaller than $2 \mathrm{~mm}$, circumscribed skin ulcerations and slightly painful lesions were observed. Later (post-infection d 15 to 25), these small lesions had coalesced to a single lesion or increased in size to $>20 \mathrm{~mm}$ across the greatest dimension. With exception of the lesion reproduced using the Treponema spp. culture, the final appearance was characterized by red coloration, sharply demarcated limit, granular moist surface, prone to bleed on manipulation, painful to touch, foul smell, and dark brown exudate accumulation. The lesion reproduced using Treponema spp. was pale red and it had poorly demarcated margins and subtle, but evident, skin alterations (Figure 4). Overall, the range of macroscopic appearances of the lesions was similar to previously published descriptions of DD (Read and Walker, 1998b). Based on clinical evaluation, original hardness of the hoof tissue was apparently regained in $24 \mathrm{~h}$ after stopping the bandaging process, although no objective measure of hoof hardness was used in the study.

\section{DISCUSSION}

Based on the proposed infection model, acute DD lesions can be induced experimentally at the dewclaw level. A previously reported study used similar experimental procedures to those described in this study and observed $100 \%$ infection rate using skin homogenates in four 4-mo-old Holstein calves (Read and Walker, 1998a), whereas the heifers used in the current study were 14 to 16 mo of age. Skin thickness increases with age (Muralidharan, 2006), which may explain the lower infection rate compared with the calf study. However, the fact that the infection has been reproduced in older animals suggests the possibility for using the infection model in cattle at an age when natural DD infection is commonly first observed in the field, and to whom treatment and prevention strategies are targeted.

The macroscopic diagnosis of DD lesions in the current study was confirmed by dark-field microscopy and PCR for Treponema spp. and by histopathological evaluation. Histopathology of the experimental DD lesions showed a consistent invasion of Treponema spp. organisms into viable tissue of the skin. Other microorganisms were found inconsistently in the bacterial mat and rarely in viable tissue. In addition, macroscopic clinical diagnosis of DD correlated with the demonstration of invasive spirochetes by Steiner silver and immunohistochemical stain evaluations.

During the current experimental infection, DD was not diagnosed in the control legs $(n=2)$. To minimize the contamination of the inoculation sites by external sources, special attention was given to the long-term and secure attachment of the rubber boots to the animals' hind legs. For that purpose, to prolong the attachment, the rubber boots were customized using nylon straps [see Figure 1(vi)]. The improved attachment mechanism of the boots allows the inclusion of larger numbers of animals into future studies while reducing labor.

This is the first attempt at standardizing an experimental infection model suitable for quantifying the effect of therapeutic or preventive measures against DD. Preconditioning of the skin by closure and maceration for at least $7 \mathrm{~d}$ before inoculation mimicked the natural exposure of cow feet to continuous moisture in deep slurry. Epidemiological studies have shown that these field conditions are strongly associated with high prevalence of PDD (Rodríguez-Lainz et al., 1996a) and that these conditions provide an adequate environment for Treponema spp. to grow (Yano et al., 2010).

Although the experimental DD lesions were found solely in the dew claw area, field DD lesions are more commonly located in the heel area (Read and Walker, 1998b). In the current infection model, differences in skin conditioning and pressure due to prolonged bandaging through the infection process could have resulted in the unsuccessful infection of the heel area. Pressure applied to the dewclaw area could contribute to an increased susceptibility to infection in this location due to more skin maceration. Additionally, DD le- 
sions were induced in the dew claws rather than in the heel area more likely due to the challenge of keeping the inoculum reservoirs at the site of infection. Specifically, the use of Super Glue at the heel area could have had a negative effect on the viability of the inoculum and the outcome of the experimental infection.

The use of tissue homogenates from naturally occurring DD infections as an inoculum produced more DD lesions than the inoculum containing Treponema spp. pure culture broth. This finding may be due to a possible synergistic effect of the presence of a wide range of microorganisms in the biopsy homogenates (Read et al., 1998; Birkett, 2008; Yano et al., 2010).

Treponema spp. culture inoculation has not been previously reported in the literature. The present study is the first attempt documented. The creation of a DD lesion with the Treponema spp. culture broth supports morphologic evidence for an important pathogenic role of Treponema spp. in DD (Read et al., 1998). Although this achievement cannot provide evidence of causality, it represents a step forward for the knowledge of the disease etiology. Further research would be needed before considering the infection with Treponema spp. culture a reliable option reproducing DD lesions experimentally.

Determination of the minimal infective dose or subtyping of the Treponema-like organisms necessary to reproduce DD lesions may improve the experimental infection model and aid in further understanding the etiology and pathogenesis of DD. In addition, the role and differences in virulence of Treponema spp. subtypes during the pathogenesis of acute DD lesions need to be studied.

Although chronic DD stages of infection have been well described as an important epidemiologic factor in the natural history of the disease (Döpfer, 1994; Read and Walker, 1998b), the final objective of the study was the reproduction of acute ulcerative DD stages defined as lesions $>20 \mathrm{~mm}$ in diameter (M2). Those M2 lesions are associated with high incidences of lameness, treatment costs, and production losses (Döpfer et al., 1997). Antibiotic treatment of the lesions immediately after an acute M2 lesion was diagnosed prevented the development of chronic lesions and further follow up was impossible, because the legs were excluded from the study. The decision to treat upon detection was originally based on animal welfare considerations at the design and Research Animal Resources Center approval of the study. Further development of the model aims at providing the conditions for developing chronic DD stages.

Field trials aimed at determining prophylactic and therapeutic effects of vaccines (Metzner et al., 2001; Ertze et al., 2006; Elliott et al., 2007), antimicrobial treatments (Holzhauer et al., 2008; Berry et al., 2010;
Teixeira et al., 2010), or other types of intervention face numerous challenges from the multifactorial pathogenesis of DD in the field. Although simultaneous considerations about the need of an appropriate sample size calculation and the availability of resources will be fundamental to the use of the described DD infection model, the authors consider the method highly valid for use in randomized controlled designs aiming at determining the efficacy of interventions. A bovine experimental infection model aims to decrease the variability of management risk factors and host factors, and presents a method for obtaining consistent DD lesions and objective clinical assessments.

\section{CONCLUSIONS}

Acute DD lesions were reproduced experimentally in Holstein heifers. The bovine DD infection model described here aids in improving the understanding of the pathogenesis of DD as an infectious hoof disease and serves as a systematic method to determine the efficacy of interventions aimed at the control of acute DD.

\section{ACKNOWLEDGMENTS}

This study was supported by Zinpro Corporation (Eden Prairie, MN). The authors thank Karl Burgi (Comfort Hoof Care Inc., Baraboo, WI), the professional animal care provided by the staff at the Livestock Laboratory (University of Wisconsin, Madison), and the time and patience of the farmers involved in the study.

\section{REFERENCES}

Berry, S. L., D. H. Read, R. L. Walker, and T. R. Famula. 2010. Clinical, histopathologic and bacteriologic findings in dairy cows with digital dermatitis (footwarts) one month after topical treatment with lincomycin hydrochloride or oxytetracycline hydrochloride. J. Am. Vet. Med. Assoc. 237:555-560.

Birkett, N. 2008. Digital dermatitis-Prevention, not cure. Int. Dairy Topics 7:19-23.

Blowey, R. W., and M. W. Sharp. 1988. Digital dermatitis in dairy cattle. Vet. Rec. 122:505-508.

Bruijnis, M. R. N., H. Hogeveen, and E. N. Stassen. 2010. Assessing economic consequences of foot disorders in dairy cattle using a dynamic stochastic simulation model. J. Dairy Sci. 93:2419-2432.

Cha, E., J. A. Hertl, D. Bar, and Y. T. Grohn. 2010. The cost of different types of lameness in dairy cows calculated by dynamic programming. Prev. Vet. Med. 97:1-8.

Cook, N. B., and K. V. Nordlund. 2009. The influence of the environment on dairy cow behavior, claw health and herd lameness dynamics. Vet. J. 179:360-369.

Döpfer, D. 1994. Epidemiological investigations of digital dermatitis on two dairy farms. MS Thesis. University of Veterinary Medicine, Hannover, Germany.

Döpfer, D., A. Koopmans, F. A. Meijer, I. Szakáll, Y. H. Schukken, W. Klee, R. B. Bosma, J. L. Cornelisse, A. J. van Asten, and A. A. ter Huurne. 1997. Histological and bacteriological evaluation of digital dermatitis in cattle, with special reference to spirochaetes and Campylobacter faecalis. Vet. Rec. 140:620-623. 
Dreher, M., D. Döpfer, T. Mumba, K. Ames, P. Sears, M. Wadell, C. Zwaagstra, and M. Plas. 1999. Clinical effect of local antibiotic treatment of digital dermatitis lesions and detection of spirochetes by means of PCR. Pages 250-251 in Proc. 32nd Ann. Conf. Am. Assoc. Bovine Pract., Nashville, TN. American Association Bovine Practitioners, Auburn, AL.

Elliott, M. K., D. P. Alt, and R. L. Zuerner. 2007. Lesion formation and antibody response induced by papillomatous digital dermatitis-associated Spirochetes in a murine abscess model. Infect. Immun. 75:4400-4408.

Ertze, R. A., D. H. Read, D. W. Hird, and S. L. Berry. 2006. Field evaluation of prophylactic and therapeutic effects of a vaccine against (papillomatous) digital dermatitis in dairy cattle on two California dairies. Bovine Pract. 40:76-82.

Ettema, J., S. Ostergaard, and A. R. Kristensen. 2010. Modelling the economic impact of three lameness causing diseases using herd and cow level evidence. Prev. Vet. Med. 95:64-73.

Evans, N. J., J. M. Brown, I. Demirkan, R. D. Murray, W. D. Vink, R. W. Blowey, C. A. Hart, and S. D. Carter. 2008. Three unique groups of spirochetes isolated from digital dermatitis lesions in UK cattle. Vet. Microbiol. 130:141-150.

Evans, N. J., J. M. Brown, I. Demirkan, P. Singh, B. Getty, D Timofte, W. D. Vink, R. D. Murray, R. W. Blowey, R. J. Birtles, C. A. Hart, and S. D. Carter. 2009. Association of unique, isolated treponemes with bovine digital dermatitis lesions. J. Clin. Microbiol. 47:689-696.

Flower, F. C., and D. M. Weary. 2009. Gait assessment in dairy cattle. Animal 3:87-95.

Gomez, A., and N. B. Cook. 2010. Time budgets of lactating dairy cattle in commercial freestall herds. J. Dairy Sci. 93:5772-5781.

Holzhauer, M., D. Döpfer, J. de Boer, and G. van Schaik. 2008. Effects of different intervention strategies on the incidence of papillomatous digital dermatitis in dairy cows. Vet. Rec. 162:41-46.

Metzner, M., W. Schutz, R. Pijl, and W. Klee. 2001. Investigations on the efficacy of herd-specific vaccines against digital dermatitis of cattle. Tierärztliche Praxis Ausgabe Grosstiere/Nutztiere $29: 345-350$.

Muralidharan, M. R. 2006. Certain factors influencing the skin thickness in exotic cattle. Tamilnadu J. Vet. Anim. Sci. 2:93-95.

Nordhoff, M., A. Moter, K. Schrank, and L. H. Wieler. 2008. High prevalence of treponemes in bovine digital dermatitis - A molecular epidemiology. Vet. Microbiol. 131:293-300.

NRC. 2001. Nutrient Requirements of Dairy Cattle. 7th rev. ed. Natl. Acad. Press, Washington, DC.

Olechnowicz, J., J. M. Jaśkowski, P. Antosik, D. Bukowska, and K. Urbaniak. 2010. Claw diseases and lameness in polish HolsteinFriesian dairy cows. Bull. Vet. Inst. Pulawy 54:93-99.

Pavlenko, A., L. Lidfors, C. Bergsten, A. Aland, and D. Arney. 2009. Claw diseases as a factor for changes in dairy cows' behaviour and milk production. Pages 135-148 in Sustainable Animal Production: The Challenges and Potential Developments for Professional Farming. A. Aland and F. Madec, ed. Wageningen Academic Publishers, Wageningen, the Netherlands.

Read, D. H., R. W. Nordhausen, and R. L. Walker. 1998. Pathogenesis of experimental papillomatous digital dermatitis (footwarts) in cattle: Bacterial morphotypes associated with early lesion development. Page 271 in Proc. 10th Int. Symp. Lameness in Ruminants, Lucerne, Switzerland.

Read, D. H., and R. L. Walker. 1994. Papillomatous digital dermatitis of dairy cattle: Pathologic findings. Pages $156-158$ in Proc. 8th Int. Sym. Dis. of Rum. Digit. Banff, Canada.
Read, D. H., and R. L. Walker. 1998a. Experimental transmission of papillomatous digital dermatitis (footwarts) in dairy cattle. Page 270 in Proc. 10th Int. Symp. Lameness in Ruminants, Lucerne, Switzerland.

Read, D. H., and R. L. Walker. 1998b. Papillomatous digital dermatitis (footwarts) in California dairy cattle: Clinical and gross pathologic findings. J. Vet. Diagn. Invest. 10:67-76.

Rodríguez-Lainz, A., D. W. Hird, T. E. Carpenter, and D. H. Read. 1996. Case-control study of papillomatous digital dermatitis in southern California dairy farms. Prev. Vet. Med. 28:117-131.

Rodriguez-Lainz, A., P. Melendez-Retamal, D. W. Hird, D. H. Read, and R. L. Walker. 1999. Farm- and host-level risk factors for papillomatous digital dermatitis in Chilean dairy cattle. Prev. Vet. Med. 42:87-97.

Scholey, R. A., W. E. R. Ollier, R. W. Blowey, R. D. Murray, and S. D. Carter. 2010. Determining host genetic susceptibility or resistance to bovine digital dermatitis in cattle. Adv. Anim. Biosci. 1:2.

Seshadri, R., G. S. A. Myers, H. Tettelin, J. A. Eisen, J. F. Heidelberg, R. J. Dodson, T. M. Davidsen, R. T. DeBoy, D. E. Fouts, D. H. Haft, J. Selengut, Q. Ren, L. M. Brinkac, R. Madupu, J. Kolonay, S. A. Durkin, S. C. Daugherty, J. Shetty, A. Shvartsbeyn, E. Gebregeorgis, K. Geer, G. Tsegaye, J. Malek, B. Ayodeji, S. Shatsman, M. P. McLeod, D. Šmajs, J. K. Howell, S. Pal, A. Amin, P. Vashisth, T. Z. McNeill, Q. Xiang, E. Sodergren, E. Baca, G. M. Weinstock, S. J. Norris, C. M. Fraser, and I. T. Paulsen. 2004. Comparison of the genome of the oral pathogen Treponema denticola with other spirochete genomes. Proc. Natl. Acad. Sci. USA 101:5646-5651.

Sogstad, Å. M., T. Fjeldaas, O. Østerås, and K. P. Forshell. 2005. Prevalence of claw lesions in Norwegian dairy cattle housed in tie stalls and free stalls. Prev. Vet. Med. 70:191-209.

Speijers, M. H. M., L. G. Baird, G. A. Finney, J. McBride, D. J. Kilpatrick, D. N. Logue, and N. E. O'Connell. 2010. Effectiveness of different footbath solutions in the treatment of digital dermatitis in dairy cows. J. Dairy Sci. 93:5782-5791.

Teixeira, A. G. V., V. S. Machado, L. S. Caixeta, R. V. Pereira, and R. C. Bicalho. 2010. Efficacy of formalin, copper sulfate, and a commercial footbath product in the control of digital dermatitis. J. Dairy Sci. 93:3628-3634.

Walker, R. L., D. H. Read, K. J. Loretz, D. W. Hird, and S. L. Berry. 1997. Humoral response of dairy cattle to spirochetes isolated from papillomatous digital dermatitis lesions. Am. J. Vet. Res. $58: 744-748$.

Walker, R. L., D. H. Read, K. J. Loretz, and R. W. Nordhausen. 1995. Spirochetes isolated from dairy cattle with papillomatous digital dermatitis and interdigital dermatitis. Vet. Microbiol. 47:343-355.

Walker, R. L., D. H. Read, S. J. Sawyer, and K. L. Loretz. 1998. Phylogenetic analysis of spirochetes isolated form papillomatous digital dermatitis lesions in cattle. Abstr. 17 in Proc. Conf. Res. Workers Anim. Dis. Chicago. Colorado State University, Fort Collins.

Wells, S. J., L. P. Garber, and B. A. Wagner. 1999. Papillomatous digital dermatitis and associated risk factors in US dairy herds. Prev. Vet. Med. 38:11-24.

Yano, T., K. K. Moe, K. Yamazaki, T. Ooka, T. Hayashi, and N. Misawa. 2010. Identification of candidate pathogens of papillomatous digital dermatitis in dairy cattle from quantitative $16 \mathrm{~S}$ rRNA clonal analysis. Vet. Microbiol. 143:352-362. 